\title{
Early radiologic and bronchoscopic changes after bronchial thermoplasty in patients with severe asthma
}

\author{
MINZHI QIU ${ }^{1,2^{*}}$, SHUSHAN WEI $^{2 *}$, ZHENGDAO LAI $^{2 *}$, PEIKAI HUANG $^{2}$, \\ ZHIQIANG WANG ${ }^{2}$, CHANGHAO ZHONG $^{3}$, YU CHEN $^{3}$, XIAOXIAN ZHANG $^{2}$, XIAOFENG LIN $^{4}$, \\ QINGSI ZENG ${ }^{4}$, KIAN FAN CHUNG ${ }^{5}$, QINGLING ZHANG ${ }^{2}$, JIAXING XIE ${ }^{2}$ and SHIYUE LI $^{3}$
}

${ }^{1}$ Department of Respiratory and Critical Care Medicine, Shenzhen Key Laboratory of Respiratory Disease, The First Affiliated Hospital of Southern University of Science and Technology, The Second Medical College of

Jinan University, Shenzhen People's Hospital, Shenzhen, Guangdong 518020; ${ }^{2}$ Department of Allergy and

Clinical Immunology, Guangzhou Institute of Respiratory Health, State Key Laboratory of Respiratory Disease, The First Affiliated Hospital of Guangzhou Medical University; ${ }^{3}$ Department of Respiratory and Critical Care Medicine, Guangzhou Institute of Respiratory Health, State Key Laboratory of Respiratory Disease, The First Affiliated Hospital of Guangzhou Medical University; ${ }^{4}$ Department of Radiology, The First Affiliated Hospital of Guangzhou Medical University, Guangzhou, Guangdong 510120, P.R. China; ${ }^{5}$ Airway Disease Section, National Heart and Lung Institute, Imperial College London and Royal Brompton and Harefield NHS Foundation Trust, London SW3 6LY, UK

Received November 19, 2019; Accepted October 6, 2020

DOI: $10.3892 /$ etm.2020.9408

\begin{abstract}
Bronchial thermoplasty (BT) is a treatment to reduce the airway smooth muscle mass by delivering radiofrequency thermal energy to the airways. BT is used in patients with severe asthma. The present study reported on cases of pneumothorax directly after BT and retrospectively analyzed early radiologic and bronchoscopic modifications after BT. The clinical data and radiologic and bronchoscopic findings of 12 patients with severe asthma who were subjected to BT between July 2014 and October 2017 were analyzed. A total of 33 chest radiographs were collected within 18-24 h after BT. Radiological abnormalities were observed in 32 radiographs as atelectasis $(53.1 \%)$, peribronchial consolidations $(84.4 \%)$,
\end{abstract}

Correspondence to: Dr Jiaxing Xie, Department of Allergy and Clinical Immunology, Guangzhou Institute of Respiratory Health, State Key Laboratory of Respiratory Disease, The First Affiliated Hospital of Guangzhou Medical University, 151 Yanjiang Road, Guangzhou, Guangdong 510120, P.R. China

E-mail: jiaxingxie@126.com

Professor Shiyue Li, Department of Respiratory and Critical Care Medicine, Guangzhou Institute of Respiratory Health, State Key Laboratory of Respiratory Disease, The First Affiliated Hospital of Guangzhou Medical University, 151 Yanjiang Road, Guangzhou, Guangdong 510120, P.R. China

E-mail: lishiyue@188.com

${ }^{*}$ Contributed equally

Key words: bronchial thermoplasty, bronchoscopy, chest radiography, pneumothorax, severe asthma pleural effusion (18.8\%), effusion in oblique fissures (3.1\%), pleural thickening (6.3\%) and pneumothorax (3.1\%). Of note, one patient suffered pneumothorax after the third BT session and underwent chest drain insertion, followed by mechanical ventilation at the intensive care unit and multiple bronchoscopic interventions, which revealed extensive phlegm plugs. A total of six patients with worsened symptoms and lobar atelectasis also required bronchoscopic intervention, which revealed that phlegm plugs occluded the bronchus in the treated lobe. No bronchoscopic intervention was required in the remaining five patients. During 16-30 days of follow-up, $95.7 \%$ of the findings on chest radiography were resolved. To the best of our knowledge, the present study reported the first case of pneumothorax following BT. Early radiologic modifications such as atelectasis and peribronchial consolidations appear common after BT. However, whether bronchoscopic intervention is required for atelectasis following BT warrants further investigation. Of note, BT should be audited and recorded in detail to ideally contribute to a framework of clinical trials to improve risk-benefit evaluations and the selection of patients likely to benefit from treatment.

\section{Introduction}

Bronchial thermoplasty (BT) is a novel therapeutic option for severe asthma. BT may significantly improve the lung function, reduce the times of acute attack and the dosage of glucocorticoids in patients with severe asthma with a predicted forced expiratory volume in $1 \mathrm{sec}\left(\mathrm{FEV}_{1}\right)$ percentage of $<60 \%$, which is safe and effective (1). Furthermore, a study demonstrated that BT may be confidently offered to patients with asthma with an $\mathrm{FEV}_{1}$ that is $30-50 \%$, prediction of no risk of more frequent or more severe adverse events and with an expected response of the same degree as that of patients with better 
lung function (2). BT primarily targets the bronchial smooth muscle ( $>3 \mathrm{~mm}$ diameter), delivering thermal energy (heated to $65^{\circ} \mathrm{C}$ ) to reduce the airway smooth muscle mass $(3,4)$. It reduces fibroblast remodelling through modifying the function of epithelial cells, particularly by reducing heat shock protein 60 secretion and subsequent signaling pathways that regulate protein arginine methyltransferase 1 expression (5). A total of 2 studies on the short-term outcomes of BT have reported that, despite its long-term effectiveness, BT may cause early radiologic modifications, including consolidation, ground-glass opacities and atelectasis $(6,7)$. However, neither of these studies presented data on bronchoscopic findings. In August 2014, a patient with severe asthma and its associated complications presented at our center. The patient had earlier undergone upper lobe atelectasis and pneumothorax followed by a third BT procedure (8). Henceforth, in our center, chest radiography and close follow-up were performed routinely in all patients after each BT session. The present study reports on a case of pneumothorax after BT procedure and retrospectively analyzed the short-term radiologic and bronchoscopic modifications in 12 patients with severe asthma after a total of 33 BT sessions.

\section{Materials and methods}

Patients. The present study retrospectively analyzed the data of 12 patients with severe asthma who were voluntarily treated with BT at Guangzhou Institute of Respiratory Health, State Key Laboratory of Respiratory Disease, The First Affiliated Hospital of Guangzhou Medical University in China between July 2014 and October 2017. The inclusion criteria were as follows: i) Subjects with asthma aged $18-65$ years; ii) severe asthma (9): Drug therapy was required for Level-4 and -5 in the past year (large dose of inhaled corticosteroids/long-acting $\beta_{2}$-agonists or leukotriene modifier/theophyline), or the systemic corticosteroid treatment lasted $\geq 50 \%$ of the time to prevent 'uncontrollable' asthma; or the 'uncontrollable' asthma still occurred in spite of the above treatment; iii) non-smoker for $\geq 1$ year (if former smoker, $<10$ pack-years total smoking history). The exclusion criteria were as follows: i) Participation in another trial within 6 weeks of the baseline period involving respiratory intervention; ii) post-bronchodilator $\mathrm{FEV}_{1}$ percentage of $<65 \%$; iii) 3 or more hospitalizations for exacerbations of asthma in the previous year; OR a history of life-threatening asthma, defined by past intubations for asthma, or intensive care unit (ICU) admission for asthma within the prior 24 months; iv) a history of recurrent lower respiratory tract infections requiring antibiotics (more than 3 in the past 12 months); v) a history of recurrent oral steroid use for asthma (4 or more pulses of oral steroids in the past 12 months); vi) known sensitivity to medications required to perform bronchoscopy (such as lidocaine, atropine and benzodiazepines); vii) known systemic hypersensitivity or contraindication to methacholine chloride or other parasympathomimetic agents; viii) acute respiratory infection; ix) other respiratory diseases including emphysema, cystic fibrosis, vocal cord dysfunction, mechanical upper airway obstruction, Churg-Strauss syndrome or allergic aspergillosis; $\mathrm{x}$ ) segmental atelectasis, lobar consolidation, significant or unstable pulmonary infiltrate, or pneumothorax confirmed by chest radiography;
Table I. Characteristics of patients with severe asthma treated by BT $(n=12)$.

\begin{tabular}{lc}
\hline Parameter & Value \\
\hline Sex (male/female) & $4 / 8$ \\
Age (years) & $48.1 \pm 2.9$ \\
Clinical status before BT & \\
Asthma duration (years) & $9.8 \pm 2.4$ \\
Number of hospital admissions & $2.1 \pm 0.4$ \\
in the previous year per patient & \\
Number of patients taking long-term & $3(25.0)$ \\
oral corticosteroid therapy & \\
Eosinophils in blood (\%) & $0.9(0.5-6.2)$ \\
Total IgE (kU/l) & $240.1 \pm 63.3$ \\
Eosinophils in induced sputum $(\%)$ & $13.5(6.5-58.5)$ \\
FEV ${ }_{1}$ pre- $\beta_{2}$-agonists predicted $(\%)$ & $56.2 \pm 10.5$ \\
FVC predicted (\%) & $93.8 \pm 20.4$ \\
MMEF predicted (\%) & $37.1 \pm 22.6$ \\
Complications & \\
Mild bronchiectasis & $2(16.7)$ \\
Nasosinusitis & $8(66.7)$ \\
Hypertension & $3(25.0)$ \\
Diabetes & $1(8.3)$ \\
OSAHS & $1(8.3)$ \\
\hline
\end{tabular}

Values are expressed as $\mathrm{n}(\%)$, the mean \pm standard deviation or median [interquartile range (25th-75th percentile)]. $\mathrm{FEV}_{1}$, forced expiratory volume in $1 \mathrm{sec}$; FVC, forced vital capacity; MMEF, maximum mid-expiratory flow; OSAHS, obstructive sleep apnea-hypopnea syndrome; BT, bronchial thermoplasty.

xi) cardiovascular disease including myocardial infarction, angina, cardiac dysfunction, cardiac dysrhythmia, conduction defect, cardiomyopathy or stroke; xii) known aortic aneurysm; xiii) significant comorbid illness including cancer, renal failure, liver disease or cerebral vascular disease; xiv) uncontrolled hypertension; xv) implanted electrical stimulation device; xvi) known coagulopathy; xvii) any other medical condition that may interfere with study participation in the opinion of the investigator. The study obtained approval from the Human Research Ethics Committee of Guangzhou Institute of Respiratory Health, State Key Laboratory of Respiratory Disease, The First Affiliated Hospital of Guangzhou Medical University (China). The patients used high doses of inhalation corticosteroids/long-acting beta-agonists and $25 \%$ were also treated with oral or systemic corticosteroids. These 12 patients underwent a total of 33 BT sessions. Oral prednisolone tablets (40 mg QD) were given three days prior to BT and three days after BT. Chest imaging evaluations included chest radiography and/or computed tomography (CT) and were routinely performed prior to BT. The demographic and clinical data of these 12 patients are presented in Table I.

$B T$. BT was performed on each patient for three sessions at intervals of at least three weeks $(10,11)$, which was supposed to ensure adequate healing of previously treated segments prior 


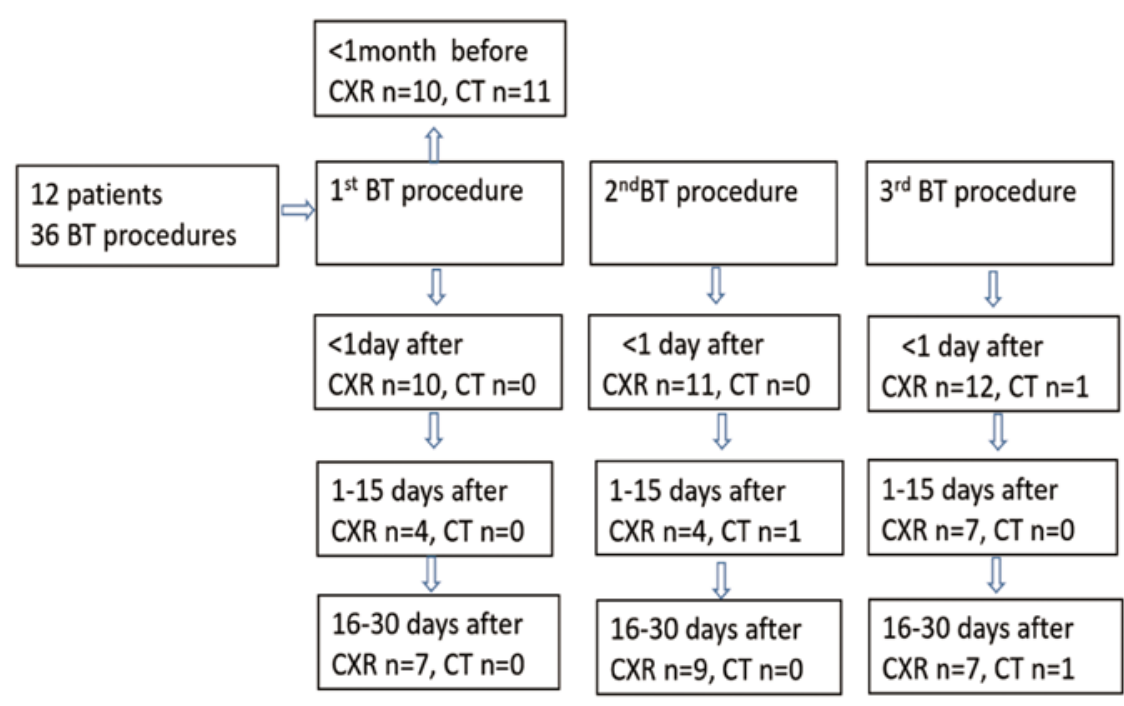

Figure 1. Flow chart of CXR examinations prior to and after BT. BT, bronchial thermoplasty; CXR, chest radiologic.

to proceeding with any further treatment. The right lower lobe was treated during the first session, the left lower lobe during the second session and the right and left upper lobes during the third session (3). The right middle lobe was not treated due to theoretical concerns of inducing right middle lobe syndrome stemming from the long and narrow lobar airway typically leading to the right middle lobe. To date, no experience in treating the right middle lobe with bronchial thermoplasty has been published (11).

Evaluations after BT. A flow chart of radiologic examinations after BT is presented in Fig. 1. Chest radiographs were obtained within 18-24 h after 33 BT sessions (chest radiography began after the third BT session in case 1 and the second BT session in case 2), as various studies indicated that radiological abnormalities were observed immediately after BT treatment $(6,7,12)$ and another study reported that a moderate temperature increase of BT causes autolysis and microvascular hemorrhage resulting in inflammation and edema after BT resembling ground-glass abnormalities (7). All possible radiologic modifications - peribronchial consolidations, atelectasis, pleural effusion, effusion in oblique fissures, pleural thickening and pneumothorax - were assessed by experienced radiologists. Symptoms such as fever, cough, chest pain, wheezing and dyspnea were also recorded from a systemic review of the clinical charts. After BT, bronchoscopic interventions were applied as necessary based on symptoms and radiologic findings. Follow-up visits were scheduled to take place 2-15 days after each BT session, as the percentage of atelectasis among 32 abnormal radiographs which were performed within 18-24 h after BT was $>50 \%$. Follow-up visits were scheduled to take place 16-30 days after each BT session, which was for the purpose of checking whether the patient was suitable for treatment with the next BT session. Radiologic findings during the two follow-up visits were also recorded.

Statistical analysis. Variables with a normal distribution are expressed as the mean \pm standard deviation and comparisons between these groups were evaluated using the indepen- dent-samples t-test. Variables with a skewed distribution were expressed as the median [interquartile range (25th-75th percentile)] and comparisons between the groups were performed using the Mann-Whitney U-test. For categorical variables, the values are expressed as $\mathrm{n}(\%)$ in each category and compared using the $\chi^{2}$ test as appropriate. $\mathrm{P}<0.05$ was considered to indicate statistical significance. All statistical analyses were performed using SPSS version 16.0 software (SPSS, Inc.).

\section{Results}

Radiologic and clinical findings during the immediate post-operative period (day 1). A total of 8 female and 4 male patients were included in the current study (mean age, 48 years). All patients underwent chest radiography $(n=10)$ or $\mathrm{CT}(\mathrm{n}=11)$ prior to the first BT session with 11 patients having no atelectasis, bronchiectasis, peribronchial consolidations, pleural effusion, effusion in oblique fissures, pleural thickening or pneumothorax.

Symptoms that occurred within $24 \mathrm{~h}$ after BT are summarized in Table II. A total of 9 patients had symptoms including cough, expectoration, tachypnea, wheezing, chest tightness, chest pain and dyspnea. None of the patients developed a fever. Within 18-24 h after BT, 12 patients underwent a total of 33 chest radiographs and $1 \mathrm{CT}$ scan. Among them, 32 radiographs exhibited modifications (Table III): Atelectasis $(\mathrm{n}=17$, 53.1\%; Fig. 2C and D and Figs. 4A and 5A), peribronchial consolidations ( $n=27,84.4 \%$; Figs. $2 \mathrm{C}-\mathrm{E}, 3 \mathrm{G}$ and $4 \mathrm{~A}$ ), pleural effusion ( $n=6,18.8 \%$; Fig. $3 \mathrm{~B}$ and $\mathrm{C})$, effusion in oblique fissures $(n=1,3.1 \%)$, pleural thickening $(n=2,6.3 \%)$ and pneumothorax $(n=1,3.1 \%)$. Only one CT scan was performed $24 \mathrm{~h}$ after BT, which revealed atelectasis in the left lower lobe (Fig. $3 \mathrm{~B}$ and $\mathrm{C}$ ). After the BT of the lower lobes, radiographs indicated early radiologic modifications in untreated lobes in three radiographs (3/20, 15.0\%; Fig. 2C). After BT of the upper lobes, radiographs exhibited radiologic modifications in the untreated lobes in six radiographs $(6 / 12,50 \%$; Figs. $2 \mathrm{E}$ and $3 \mathrm{G})$, which were manifested mainly as peribronchial consolidations in the left lower lobe $(n=5)$. 
Table II. Clinical data for the bronchoscopic treatment and no-treatment groups after bronchial thermoplasty.

A, Prior to the first BT session

\begin{tabular}{|c|c|c|c|}
\hline Parameter & $\begin{array}{l}\text { Bronchoscopic treatment } \\
\text { group }(n=7)\end{array}$ & $\begin{array}{l}\text { Non-bronchoscopic treatment } \\
\text { group }(n=5)\end{array}$ & P-value \\
\hline Sex (male/female) & $3 / 4$ & $1 / 4$ & 0.428 \\
\hline Age (years) & $49.4 \pm 12.9$ & $42.8 \pm 11.1$ & 0.376 \\
\hline BMI $\left(\mathrm{kg} / \mathrm{m}^{2}\right)$ & $24.7 \pm 3.1$ & $22.2 \pm 2.1$ & 0.156 \\
\hline Disease course (years) & $11.6 \pm 9.2$ & $7.4 \pm 7.3$ & 0.421 \\
\hline Smokers & 2 & 1 & 0.746 \\
\hline Times of hospitalization in the past year & $1.4 \pm 1.0$ & $3.0 \pm 1.6$ & 0.058 \\
\hline Number of patients with underlying disease & 5 & 4 & 0.746 \\
\hline Nasosinusitis & 4 & 4 & \\
\hline Mild bronchiectasis & 2 & 0 & \\
\hline Hypertension & 3 & 0 & \\
\hline Diabetes & 1 & 0 & \\
\hline OSAHS & 1 & 0 & \\
\hline Blood WBC count $(109 / 1)$ & $8.1 \pm 2.0$ & $10.5 \pm 3.7$ & 0.243 \\
\hline Percentage of neutrophils in blood (\%) & $58.8(53.8-60.9)$ & $66.9(55.4-70.2)$ & 0.570 \\
\hline Percentage of eosinophils in blood (\%) & $1.35(0.2-5.6)$ & $0.9(0.5-8.1)$ & 1.000 \\
\hline Eosinophils in induced sputum (\%) & $13.0(11.4-51.8)$ & $36.5(1.8-58.3)$ & 0.806 \\
\hline Neutrophils in induced sputum (\%) & $82.0(38.9-85.4)$ & $58.0(33.8-86.8)$ & 0.808 \\
\hline $\operatorname{TIgE}(\mathrm{KU} / \mathrm{l})$ & $207.0 \pm 178.8$ & $286.4 \pm 282.1$ & 0.561 \\
\hline FVC predicted $(\%)$ & $82.7 \pm 18.7$ & $109.5 \pm 9.9$ & 0.010 \\
\hline $\mathrm{FEV}_{1}$ pre- $\beta_{2}$-agonists predicted (\%) & $65.1 \pm 22.0$ & $87.8 \pm 9.0$ & 0.038 \\
\hline FEV1/FVC (\%) & $64.6 \pm 11.2$ & $69.4 \pm 11.5$ & 0.489 \\
\hline MMEF predicted (\%) & $32.5 \pm 21.4$ & $43.6 \pm 25.1$ & 0.429 \\
\hline
\end{tabular}

B, $24 \mathrm{~h}$ after three BT sessions

\begin{tabular}{|c|c|c|c|}
\hline Parameter & $\begin{array}{l}\text { Bronchoscopic treatment } \\
\text { group }(n=7)\end{array}$ & $\begin{array}{l}\text { Non-bronchoscopic treatment } \\
\text { group }(n=5)\end{array}$ & P-value \\
\hline \multicolumn{4}{|l|}{ Symptoms/complaints } \\
\hline Cough & 11 & 3 & 0.049 \\
\hline Expectoration & 11 & 3 & 0.049 \\
\hline Tachypnea & 4 & 0 & 0.077 \\
\hline Wheezing & 6 & 0 & 0.025 \\
\hline Chest tightness & 4 & 0 & 0.077 \\
\hline Chest pain & 1 & 0 & 0.398 \\
\hline Dyspnea & 1 & 0 & 0.398 \\
\hline Radiologic findings & 17 & 15 & \\
\hline Lobar atelectasis & $11(64.7)$ & $0(0)$ & $<0.001$ \\
\hline Segmental atelectasis & $0(0)$ & $6(40.0)$ & 0.004 \\
\hline Peribronchial consolidations & $13(76.5)$ & $14(93.3)$ & 0.197 \\
\hline Average hospital stays for 3 BT sessions & $4.4 \pm 1.8$ & $2.2 \pm 0.7$ & 0.017 \\
\hline
\end{tabular}



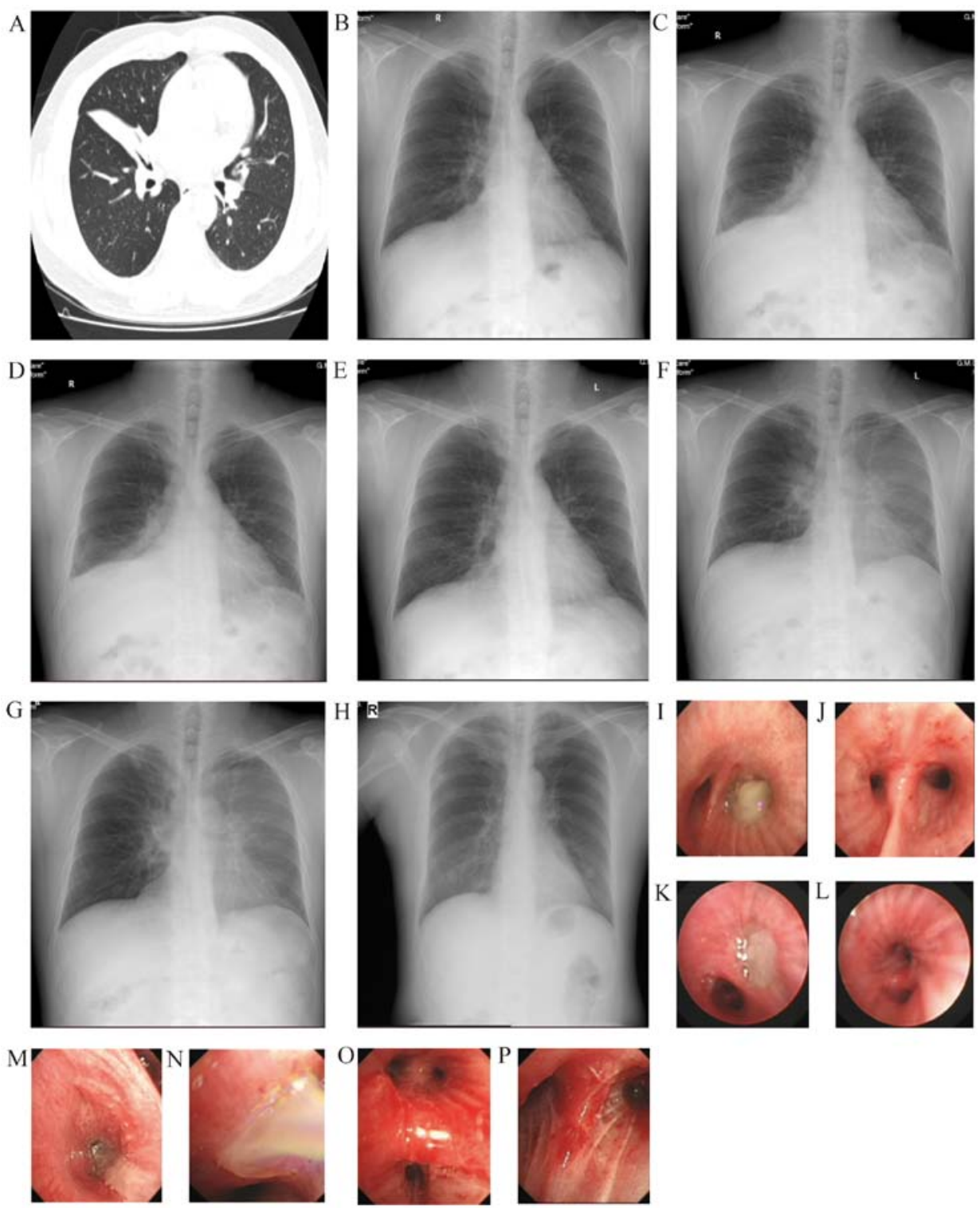

Figure 2. Representative images of a 45-year-old male patient. (A) At 46 days prior to the first BT session, atelectasis was observed in the right middle lung. (B) At four days prior to the first BT session, slight infiltrates were present in the right middle lung. (C) Within $24 \mathrm{~h}$ after the first BT session, atelectasis was observed in the right middle lobe, along with peribronchial consolidations in the right lower lung. (D) Within $24 \mathrm{~h}$ after the second BT session, atelectasis and peribronchial consolidations were present in the left lower lobe. (E) Within $24 \mathrm{~h}$ after the third BT session, peribronchial consolidations were observed in the left lower lung. (F) Within five days after the third BT session, peribronchial consolidations in the left lung (upper and lower lobes) had increased. (G) Within 16 days after the third BT session, peribronchial consolidations in the left lung were the same as before. $(\mathrm{H})$ Within five months after the third BT session, the results were normal. At 4 days after the first BT session, (I) sticky phlegm occluded the right lower lobe bronchus and (J) the right lower lobe bronchus became patent after treatment. At 2 days after the second BT session, (K) the left lower lobe bronchus was occluded by yellowish phlegm and (L) the basal and dorsal segments of the lower lobe became patent. At 1 day after the third BT session, numerous white sticky phlegm plugs in (M) the right upper lobe and (N) left upper lobe. Congestion and swelling of bronchial mucosa in $(\mathrm{O})$ left and $(\mathrm{P})$ right upper lobes were present after treatment. Chest CTs are demonstrated in (A). $\mathrm{X}$-rays are presented in (B, C-G). Bronchoscopy images are demonstrated in (I-P). BT, bronchial thermoplasty.

Table III. Early radiological findings on the day after bronchial thermoplasty.

\begin{tabular}{lc}
\hline Item & $\mathrm{N}(\%)$ \\
\hline Peribronchial consolidations & $27(84.4)$ \\
Atelectasis & $17(53.1)$ \\
Pleural effusion & $6(18.8)$ \\
Oblique effusion & $1(3.1)$ \\
Pleural thickening & $2(6.3)$ \\
Pneumothorax & $1(3.1)$ \\
\hline
\end{tabular}

\section{Radiological and bronchoscopic findings after BT}

Follow-up visits 2-15 days after BT. A total of seven patients developed tachypnea, chest tightness, chest pain, wheezing or dyspnea after a total of nine BT sessions, and all of them had atelectasis, located in the treated lobes in six cases (Figs. 3-5) and in untreated lobe in one case (Fig. 2C). A total of 23 bronchoscopic examinations were required in these patients (bronchoscopic intervention group), which revealed that phlegm plugs occluded the bronchus in the treated lobe, whereas there was a small amount of white phlegm in the bronchus of the untreated lobe. Bronchoscopic aspiration, fragmentation and washing were applied to remove the secretions and plugs. At 

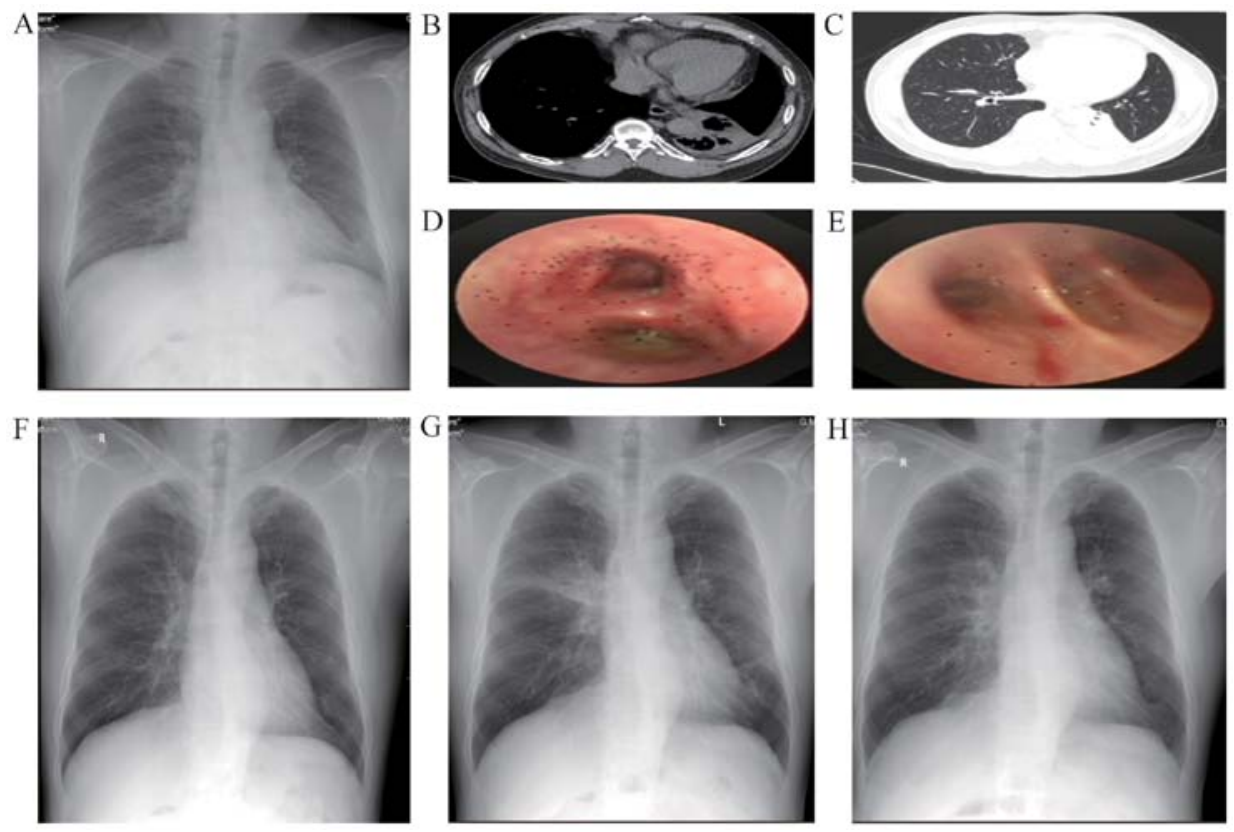

Figure 3. Representative images of a 50-year-old male. (A) At three days prior to the first BT session, the appearance was normal. Within $24 \mathrm{~h}$ after the second BT session, pleural effusion (B) and left lower lung atelectasis (C) of the left lung were observed. (D) At three days after the second BT session, yellow viscous secretions were present in the left lower lobe. (E) At 10 days after the second BT session, yellow viscous secretions in the left lower lobe were observed. (F) At 15 days after the second BT session, atelectasis had basically disappeared. (G) Within $24 \mathrm{~h}$ after the third BT session, peribronchial consolidations in the right upper lobe and left lower lobe were present. (H) At three days after the third BT session, radiological resolution in the right upper lobe and left lower lobe was observed. X-rays are presented in (A, F, G and H). Chest CTs are demonstrated in (B and C). Bronchoscopy images are presented in (D and E). BT, bronchial thermoplasty.
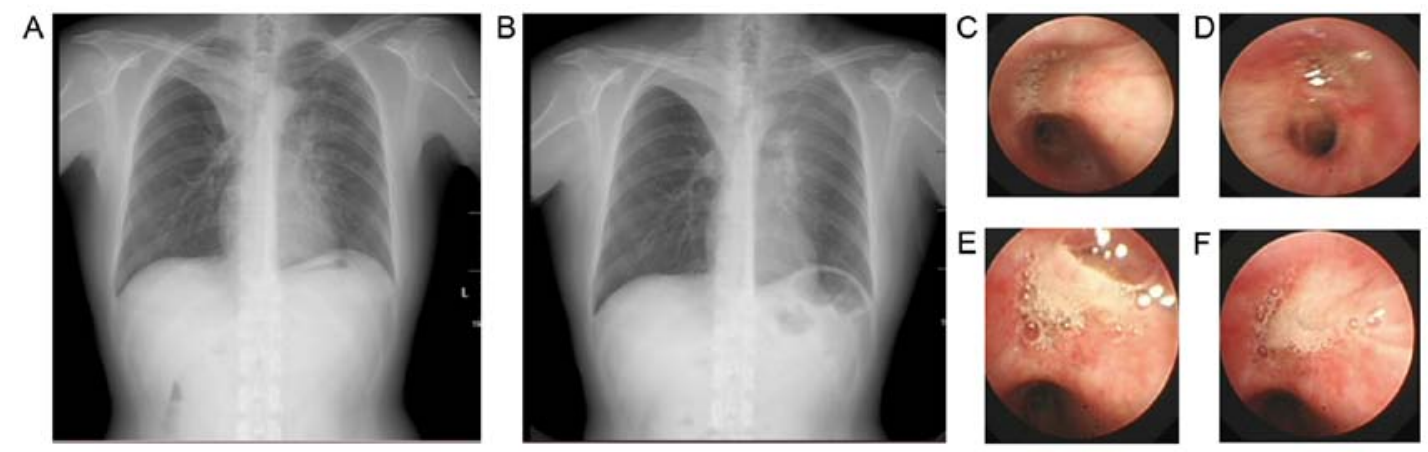

Figure 4. Representative images of a 49-year-old female. (A) Within $24 \mathrm{~h}$ after the third BT session, atelectasis and peribronchial consolidations in the right upper lobe and in the left lung were observed. (B) At three days after the third BT session, atelectasis and peribronchial consolidations were present in both upper lobes. At one day after the third BT session, sputum plugs blocked the apical and posterior segments of (C) the right upper lobe and (D) left upper and lingual lobes. (E and F) At four days after the third BT session, sputum plugs blocked (E) the right upper lobe and (F) the left upper and lingual lobes. X-ray images are presented in (A and B). Bronchoscopy images are presented in (C-F). BT, bronchial thermoplasty.

the same time, oral/systemic glucocorticoids and expectorants were given to counteract airway inflammation and cough up mucus. There were no positive results for microorganisms. In one patient, bronchoscopic aspiration was required after every BT session (Fig. 2K-P). In two patients, bronchoscopy was required after the second BT session to remove phlegm plugs, requiring five bronchoscopic sessions (Fig. 3D and E). In four patients, bronchoscopy was required after the third BT session to remove phlegm plugs. Hence, a total of 10 chest radiographs were obtained from these seven patients 2-15 days after BT sessions. Atelectasis was resolved in 5 patients $(5 / 7,71.4 \%$; Figs. $3 \mathrm{~F}$ and $4 \mathrm{E}$ ), peribronchial consolidations decreased or were resolved in 4 patients $(4 / 7,57.1 \%$; Figs. $3 \mathrm{H}$ and $4 \mathrm{E})$ and pneumothorax was resolved in 1 patient (1/1, 100\%; Fig. 5E).
The remaining five patients (including four who developed segmental atelectasis within 18-24 h after BT and one without atelectasis after BT) did not exhibit any obvious pulmonary symptoms after BT and therefore did not undergo bronchoscopy (non-bronchoscopic intervention group). All of these patients were given ambroxol, myrtol standardized enteric-coated soft capsules and inhaled medications. A total of five chest radiographs were assessed at this follow-up, which indicated that the segmental atelectasis resolved in three radiographs and peribronchial consolidations decreased or disappeared in four radiographs $(4 / 5,80 \%)$.

The clinical data of the bronchoscopic and non-bronchoscopic intervention groups are presented in Table II. Baseline pulmonary function, average hospital stays for three BT 

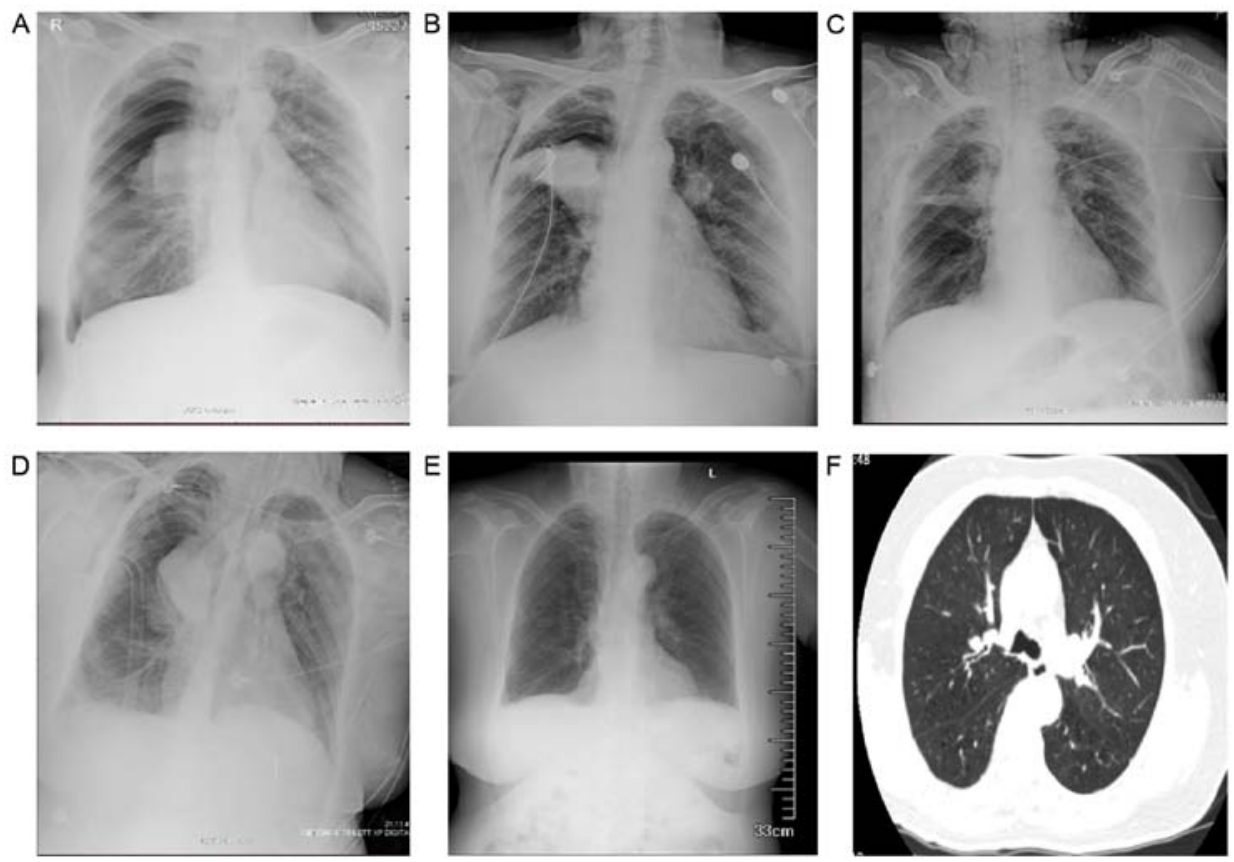

Figure 5. Representative images of a 65 -year-old female. (A) Within $24 \mathrm{~h}$ after the third BT session, right upper lobe atelectasis and pneumothorax in the right upper lobe were observed (50-60\% collapsed). (B) At $30 \mathrm{~h}$ after the third BT session, the right lung was 35\% collapsed and atelectasis of the right upper lobe was resolved. (C) At 4 days after the third BT session, pneumothorax was resolved and upper right lobe atelectasis was relieved. (D) At five days after the third BT session, right-sided pneumothorax was worsened and consolidation and atelectasis in the right upper lobe were more obvious. (E) At 10 days after the third BT session, radiological resolution in the right upper lobe was achieved. (F) At 27 days after the third BT session, observations were normal. X-rays are presented in (A-E). A chest CT is demonstrated in (F). BT, bronchial thermoplasty.

sessions and post-operative cough/expectoration, wheezing and radiologic modifications (bronchial lobar or segmental atelectasis) were significantly poorer in the bronchoscopic intervention group.

Follow-up visits 16-30 days after BT. No bronchoscopy was required. A total of 23 chest radiographic assessments revealed that $95.7 \%(22 / 23)$ of the radiographic modifications observed at the previous examinations were markedly decreased or disappeared. One exception was peribronchial consolidations in the left lung (Fig. 2G).

Bronchoscopy and radiologic findings after BT in three representative cases. A 66-year-old female retired worker presented with a BMI of $26.2 \mathrm{~kg} / \mathrm{m}^{2}$, an Asthma Control Test score of 18, Asthma Control Questionnaire score of 1 and Mini-Asthma Quality of Life Questionnaire score of 5. The patient was a non-smoker and had hypertension for 11 years and abnormal glucose tolerance. Furthermore, the patient had a 26-year history of asthma. At two months prior to BT, the patient's lung function exhibited severe, mixed ventilation dysfunction $\left[\mathrm{FEV}_{1} \%\right.$ predicted, $34.0 \% ; \mathrm{FEV}_{1} /$ forced vital capacity (FVC), 49.8\%] and chest CT was normal. The patient did not have any symptoms after the first or second BT session, and accordingly, no radiologic examination was performed. At $12 \mathrm{~h}$ after the third BT session, the patient had symptoms of wheezing, dyspnea and oxygen desaturation (89\%), which were treated with intravenous methylprednisolone. At $15 \mathrm{~h}$ after the procedure, the symptoms of severe wheezing, chest tightness and dyspnea continued to worsen with desaturation [saturated oxygen pressure $\left(\mathrm{SpO}_{2}\right), 88 \%$ under medium-flow oxygen inhalation]. Bedside chest radiography revealed atel- ectasis in the right upper lobe and pneumothorax surrounding the right upper lobe (50-60\% collapsed) (Fig. 5A). After chest drain insertion, the above symptoms remained and type II respiratory failure $[\mathrm{pH} 7.281$; partial carbon dioxide pressure $\left(\mathrm{PaCO}_{2}\right), 51.9 \mathrm{mmHg} ; \mathrm{PaO}_{2}, 75.1 \mathrm{mmHg}$ ] occurred. The patient was transferred to the ICU for noninvasive ventilation. Bronchoscopy revealed swelling of the bronchial mucosa and plugs blocking the bronchi of the left and right upper lobes, which were removed by biopsy forceps and washing. At four days after the third BT procedure, the chest radiograph indicated resolved pneumothorax and relieved upper right lobe atelectasis (Fig. 5C). The next day, dyspnea had worsened $\left(\mathrm{SpO}_{2}, 82 \%\right)$. Radiography indicated a worsened right-sided pneumothorax, as well as more obvious consolidation and atelectasis in the right upper lobe (Fig. 5D). The results of the blood gas analysis were as follows: $\mathrm{pH} 7.524 ; \mathrm{PaO}_{2}, 61 \mathrm{mmHg}$; $\mathrm{PaCO}_{2}, 29.5 \mathrm{mmHg}$; and fraction of inspired oxygen, 90\%). After endotracheal intubation and mechanical ventilation, bronchoscopy was performed daily to remove plugs, along with administration of broad-spectrum antibiotics. After four days, the intubation and thoracic drainage tube were successfully withdrawn and sequential noninvasive mechanical ventilation was maintained. On day 10, after BT, radiography indicated no atelectasis (Fig. 5E). On day 27, the patient's radiographs were basically normal (Fig. 5F).

A 49-year-old male patient with a BMI of $26.8 \mathrm{~kg} / \mathrm{m}^{2} \mathrm{had}$ a 10 -year history of asthma. The patient had a 20 pack-year smoking history but had not smoked for two years. Furthermore, the patient obstructive sleep apnea, hypertension and type 2 diabetes. At one week prior to BT, lung function tests revealed severe obstructive ventilation dysfunction $\left(\mathrm{FEV}_{1}\right.$ predicted percentage, 49.7\%; $\mathrm{FEV}_{1} / \mathrm{FVC}, 55.8 \%$ ). Radiography revealed 
left lower lobe atelectasis and CT indicated left lower lung atelectasis and pleural effusion of left lung after the second BT session (Fig. 3B and C). The complete blood count (CBC) was normal. The patient had symptoms of expectoration and wheezing, and was treated with intravenous methylprednisolone and non-invasive ventilation. The patient required three bronchoscopic interventions, which revealed plugs at the left lower lobe (Fig. 3D and E). On day 15 after BT, radiography indicated that atelectasis had almost disappeared (Fig. 3F). On day 47, the patient underwent a third BT session. Radiography performed within $24 \mathrm{~h}$ after this session revealed peribronchial consolidations in the right upper lobe and left lower lobe (Fig. 3G). At the same time, the patient had symptoms of wheezing, which were markedly alleviated after frequent nebulized intravenous methylprednisolone treatment. After three days, radiography indicated improvement (Fig. 3H).

A 46-year-old male presented with a 5-year history of asthma and allergic rhinitis. The patient had a 1 pack-year smoking history but had not smoked for seven years. In 2012, the patient underwent tracheal intubation at our hospital due to a severe asthma attack. The patient's lung function recorded in the previous last year indicated severe, mixed ventilation dysfunction $\left(\mathrm{FEV}_{1}\right.$ predicted percentage, $41.9 \%$; $\mathrm{FEV}_{1} / \mathrm{FVC}$, 49.7\%). Prior to the first BT session, lung function tests revealed moderate obstructive ventilatory dysfunction $\left(\mathrm{FEV}_{1}\right.$ predicted percentage, $61.9 \% ; \mathrm{FEV}_{1} / \mathrm{FVC}, 65.8 \%$ ). Radiography performed within $24 \mathrm{~h}$ after the first BT session revealed atelectasis in the right middle lobe and peribronchial consolidations in the right lower lobe (Fig. 2C). The $\mathrm{CBC}$ revealed a white blood cell count (WBC) of $23.55 \times 109 / 1$ with $82.9 \%$ neutrophils. The patient complained of a productive cough with yellow sticky phlegm. The patient was given intravenous moxifloxacin, methylprednisolone and frequent nebulized treatments. Bronchoscopy indicated that yellow, sticky phlegm occluded the right lower lobe bronchus (Fig. 2I). After bronchoscopic washing and ambroxol lavage, the right lower lobe bronchus became clear (Fig. 2J) and the symptoms markedly diminished. The patient underwent a second BT session 43 days later. Within $24 \mathrm{~h}$, radiography revealed atelectasis and peribronchial consolidations in the left lower lobe (Fig. 2D). The CBC revealed a WBC of $18.6 \times 10^{\%} / 1$ with $81.2 \%$ neutrophils and $0.2 \%$ eosinophils. Bronchoscopy indicated that the left lower lobe bronchus was occluded by yellowish phlegm (Fig. 2K). After 76 days, the patient underwent a third BT session and on the following day, radiography indicated peribronchial consolidations in the left lower lobe (Fig. 2E). Bronchoscopy revealed that bronchi in the right and left upper lobes were occluded by numerous white, sticky phlegm plugs (Fig. 2M-P). After five days, radiography suggested that peribronchial consolidations in the left lung (upper and lower lobes) had increased (Fig. 2F) and the patient was still wheezing. After one week of administering intravenous moxifloxacin, methylprednisolone and frequent nebulized treatments, the wheezing diminished. At 5 months thereafter, the patient's radiography results were normal (Fig. 2H).

\section{Discussion}

In a previous study by our group (8), a patient who suffered from pneumothorax directly after BT was briefly described.
To the best of our knowledge, this was the first case that required intubation and mechanical ventilation in an ICU after BT. In the present study, this case was described in detail by displaying the chest radiologic modifications. The collapse of the upper lobe due to bronchus occlusion and excess traction on pleura was the major cause of the localized pneumothorax. Further possible causes may be old age, poor basic pulmonary function and underlying diseases. Recently, Funatsu et al (13) reported on a case of pulmonary cyst and pneumothorax after BT. Similar to our case, their patient experienced hypoxemia and complete bilateral upper lobe atelectasis after the third BT session. However, their case featured the sudden emergence of a pulmonary cyst in the right middle lobe associated with pneumothorax on post-operative day 6 . They explained that pneumothorax was caused by the rupture of the pulmonary cyst that had been formed during the pulmonary function test after BT.

To the best of our knowledge, the present study was the first to evaluate the short-term lung conditions following BT based on both chest radiography and bronchoscopy. Abnormal radiologic findings at 18-24 $\mathrm{h}$ after each BT session included atelectasis, peribronchial consolidation, opacities, pleural effusion, effusion in oblique fissures, pleural thickening and pneumothorax. Bronchoscopic abnormalities at one day after each BT session included sticky phlegm and mucus plugging in the corresponding bronchi. In most cases, these radiologic modifications were markedly decreased or had disappeared within one month after the use of oral/systemic glucocorticoids, expectorants and/or bronchoscopic interventions.

Recently, Debray et al (6) analyzed early CT modifications induced by BT in 13 patients who had undergone a total of $27 \mathrm{BT}$ sessions. The chest CT performed on the day after BT revealed pulmonary peribronchial consolidations and ground-glass opacities in all treated lobes, as well as atelectasis (lobar and segmental). The percentage of abnormal CT modifications was 68\%. d'Hooghe et al (7) analyzed 34 chest radiographs and 16 chest CT scans of 12 severe asthmatic patients directly after BT. Approximately $91 \%$ of the radiographs exhibited modifications, including peribronchial consolidations (97\%) and atelectasis (29\%). No bronchoscopic intervention was required. The follow-up CT scan after six months indicated resolution of the changes. d'Hooghe et al (7) performed the chest X-ray within $<5 \mathrm{~h}$ after BT and Debray et al (6) performed the CT scan on the day after BT. In the present study, the chest X-ray was performed at 18-24 $\mathrm{h}$ after each BT session. In line with the results of d'Hooghe et al (7) and Debray et al (6), similar chest radiologic modifications were observed the present study. These two previous studies and also the present study indicated that peribronchial consolidations are the most frequent modifications shortly after BT. Atelectasis at 18-24 h after BT occurred 53\% of cases in the present patient cohort, while Debray et al (6) and d'Hooghe et al (7) reported an incidence of 68 and 38\%, respectively. Debray et al (6) reported that CT changes were not associated with respiratory symptoms. In comparison, the patients with severe chest radiological modifications of the present study had more symptoms.

Debray et al (6) reported that opacities occurred in 12 untreated lobes in one-third of patients on the day after BT. The BT-untreated lobes involved the middle lobe $(n=5)$ and the 
right lower lobe $(n=4)$. An explanation for this may be the diffusion of heat shock of BT along the bronchial wall and extension of heat shock through fissures (11). d'Hooghe et al (7) reported that $31 \%$ of the chest CTs had ground-glass opacities in the neighboring non-BT-treated lobe. However, no abnormalities were detected in the non-BT-treated right middle lobe. The flow of mucosal blood and/or mucus runs down from the BT-treated upper lobes to untreated lower lobes, which may be a reasonable explanation for the radiological abnormalities being located only in the non-BT-treated lower lobes in all of the patients of that study. In the present study, $15 \%$ of chest radiographs exhibited early modifications in the non-treated right upper lobes after BT in the left lower lobes. However, after BT of the right and left upper lobes, $50 \%$ of chest radiographs exhibited abnormalities (mainly peribronchial consolidations) in untreated lower lobes. In addition, bronchoscopic modifications in untreated lobes generally included small amounts of white secretions in the bronchus and swelling of the mucous membranes.

In the present study, 7 of the 12 patients required bronchoscopic intervention after BT. The bronchoscopic abnormality (mucus plugging) corresponded to imaging abnormalities of the same lung lobe. The bronchoscopic intervention group exhibited severe eosinophilic inflammation (median sputum eosinophil ratio, 13\%) which may contribute to the development of mucus plugging after BT. However, the non-bronchoscopic group exhibited severe eosinophilic inflammation (median sputum eosinophil ratio, 36.5\%) and had milder symptoms and imaging abnormalities after BT. Debray et al (6) and d'Hooghe et al (7) did not perform any induced sputum analysis. Therefore, it is not known whether there was an obvious eosinophilic airway inflammation in their patients presenting with atelectasis after BT. It remains elusive whether there is an association between eosinophilic airway inflammation and mucus plugging of the airway after BT. Compared with the non-bronchoscopic intervention group, the patients who required bronchoscopic intervention had poorer lung function (pre- $\beta_{2}$-agonist mean $\mathrm{FEV}_{1}$ predicted percentage, $65.1 \%$ ) and were more likely to develop lung lobar atelectasis (not segmental atelectasis), with their lobar bronchi occluded by phlegm plugs. However, Debray et al (6) reported that the $\mathrm{FEV}_{1}$ predicted percentage in 13 patients was $60.5 \%$ and these patients had a longer duration of asthma and more hospitalizations during the past year. No bronchoscopic intervention was required for these patients, which may be explained by their relatively younger age (average, 40.8 years). Similarly, d'Hooghe et al (7) reported that no bronchoscopic intervention was required for their patients. Facciolongo et al (14) reported on a case of recurrent lobar collapse occurring a few hours after two BT sessions. The patient had acute respiratory failure and required bronchoscopic removal of plugs. In the present study, seven patients needed bronchoscopic intervention, but the intervention was unable to relieve the radiological abnormality for one patient after the third BT session. Further well-designed controlled studies are warranted to determine whether bronchoscopic intervention is required in patients developing lobar atelectasis following BT.

In summary, to the best of our knowledge, the present study reported the first case of pneumothorax following BT in need of intubation. Furthermore, there was a high incidence of early radiologic abnormalities (i.e., mainly atelectasis and peribronchial consolidations) after BT. Bronchoscopy revealed occlusion of the bronchus by phlegm plugs at the corresponding sites. However, whether bronchoscopic intervention is needed for atelectasis following BT requires further investigation. Besides this, BT should be audited and recorded in detail and ideally contribute to a framework of clinical trials in order to improve risk-benefit evaluations and the selection of patients likely to benefit from treatment.

\section{Acknowledgements}

The authors thank Dr Jiang Mei (Guangzhou Institute of Respiratory Health, State Key Laboratory of Respiratory Disease, The First Affiliated Hospital of Guangzhou Medical University) for her assistance with the statistical analysis.

\section{Funding}

This research was funded by the Natural Science Foundation of Guangdong Province (grant no. 2019A1515010622), Professor K.F. Chung's Visiting Professor Project of Guangzhou Institute of Respiratory Health (grant no. 500102010501069001) and the National Natural Science Foundation of China (grant no. 81770017).

\section{Availability of data and materials}

All data generated or analyzed during this study are included in this published article.

\section{Authors' contributions}

JX and SL conceived and designed the current study. QZ, SL, MQ, SW and XZ provided administrative support. MQ, SW, $\mathrm{XZ}$ and ZL searched the literature. JX, PH, ZW collected and collated data. MQ, SW, ZL, CZ, YC, XL, QZ and KC analyzed and interpreted the data. All authors wrote the manuscript and all authors read and approved the final manuscript.

\section{Ethics approval and consent to participate}

The study complied with the Declaration of Helsinki and was approved by the Institutional Review Board Ethics Committee of the First Affiliated Hospital of Guangzhou Medical University. Written informed consent was obtained from each patient.

\section{Patient consent for publication}

The patients consented to the publication of their data and images in the present study.

\section{Competing interests}

The authors declare that they have no competing interests.

\section{References}

1. Long F, Zhong D, Huang WT, Long L, Hu FB, Fu P and Hu SY: Analysis of the safety and efficacy of bronchial thermoplasty for severe asthma with the first second forced expiratory volume $(\mathrm{FEV}(1))$ as a percentage of the predicted value $(\mathrm{FEV}(1) \%$ pred $)<60$. Zhonghua Yi Xue Za Zhi 100: 2023-2027, 2020 (In Chinese). 
2. Langton D, Ing A, Fielding D, Hersch N, Sha J, Plummer V and Thien F: Safety and Effectiveness of Bronchial Thermoplasty When FEV1 Is Less Than 50. Chest 157: 509-515, 2020.

3. Pavord ID, Cox G, Thomson NC, Rubin AS, Corris PA, Niven RM, Chung KF and Laviolette M; RISA Trial Study Group: Safety and efficacy of bronchial thermoplasty in symptomatic, severe asthma. Am J Respir Crit Care Med 176: 1185-1191, 2007.

4. Dombret MC, Alagha K, Boulet LP, Brillet PY, Joos G, Laviolette M, Louis R, Rochat T, Soccal P, Aubier M, et al: Bronchial thermoplasty: A new therapeutic option for the treatment of severe, uncontrolled asthma in adults. Eur Respir Rev 23: 510-518, 2014.

5. Sun Q, Fang L, Roth M, Tang X, Papakonstantinou E, Zhai W, Louis R, Heinen V, Schleich FN, Lu S, et al: Bronchial thermoplasty decreases airway remodelling by blocking epithelium-derived heat shock protein-60 secretion and protein arginine methyltransferase-1 in fibroblasts. Eur Respir J 54: 1900300, 2019.

6. Debray MP, Dombret MC, Pretolani M, Thabut G, Alavoine L, Brillet PY, Taillé C, Khalil A, Chanez P and Aubier M: Early computed tomography modifications following bronchial thermoplasty in patients with severe asthma. Eur Respir J 49: $1601565,2017$.

7. d'Hooghe JNS, van den Berk IAH, Annema JT and Bonta PI: Acute Radiological Abnormalities after Bronchial Thermoplasty: A Prospective Cohort Trial. Respiration 94: 258-262, 2017.

8. Zhang Q, Zhang X, Xie J, Qiu R, Chen Y, Huang Z, He Y, Xian M, Li J and Li S: Bronchial thermoplasty in the treatment of severe asthma. Zhonghua Jie He He Hu Xi Za Zhi 39: 183-188, 2016 (In Chinese).
9. Chung KF, Wenzel SE, Brozek JL, Bush A, Castro M, Sterk PJ, Adcock IM, Bateman ED, Bel EH, Bleecker ER, et al: International ERS/ATS guidelines on definition, evaluation and treatment of severe asthma. Eur Respir J 43: 343-373, 2014.

10. Cox G, Thomson NC, Rubin AS, Niven RM, Corris PA, Siersted HC, Olivenstein R, Pavord ID, McCormack D, Chaudhuri R, et al; AIR Trial Study Group: Asthma control during the year after bronchial thermoplasty. N Engl J Med 356: 1327-1337, 2007.

11. Mayse M, Laviolette M, Rubin A, Lampron N, Simoff M, Duhamel D, Musani AI, Yung RC and Mehta AC: Clinical pearls for bronchial thermoplasty. J Bronchology Interv Pulmonol 14: 115-123, 2007.

12. d'Hooghe JNS, Bonta PI, van den Berk IAH and Annema JT: Radiological abnormalities following bronchial thermoplasty: Is the pathophysiology understood? Eur Respir J 50: 1701537, 2017.

13. Funatsu A, Kobayashi K, Iikura M, Ishii S, Izumi S and Sugiyama H: A case of pulmonary cyst and pneumothorax after bronchial thermoplasty. Respirol Case Rep 6: e00286, 2017.

14. Facciolongo N, Menzella F, Lusuardi M, Piro R, Galeone C, Castagnetti C, Cavazza A, Carbonelli C, Zucchi L and Salsi PP: Recurrent lung atelectasis from fibrin plugs as a very early complication of bronchial thermoplasty: A case report. Multidiscip Respir Med 10: 9, 2015.

(i) $\Theta$ This work is licensed under a Creative Commons

Attribution-NonCommercial-NoDerivatives 4.0 International (CC BY-NC-ND 4.0) License. 